\title{
Role of HPV in Oral Cancer
}

\section{Smitha T*}

Professor, Department of Oral Pathology, Rajiv Gandhi University Health Sciences, India

*Corresponding Author: Smitha T, Professor, Department of Oral Pathology, Rajiv Gandhi University Health Sciences, India.

DOI: 10.31080/ASDS.2020.04.0899
Received: June 08, 2020

Published: July 30, 2020

(C) All rights are reserved by Smitha T.

\begin{abstract}
Oral cancer is one of the major health issues in India. The traditional risk factors or oral cancer include pan chewing, tobacco and alcohol use. However, Human papillomavirus (HPV) 16 is now being implicated causing oral cancer. Until recent times, the research on oral cancer has focused on factors like genetics, evolving lifestyle and environmental factors such as pollution. The possible etiological role of HPV16 and 18 in oral oncogenesis needs to be addressed. Additionally, there are many aspects of HPV in causation of oral cancer that still needs to be understood and addressed. The following chapter focuses on types and role of HPV, its pathogenesis within the oral cavity, and prognosis in case of HPV-positive cancer cases. The chapter also includes an update on the diagnostic tests, with highlight on various specific biomarkers, and the treatments developed to contain the HPV-positive cancer cases. Current research areas and future perspectives have been covered in this chapter which can be taken up to advance the research on HPVpositive oral cancer.
\end{abstract}

Keywords: Human Papillomavirus (HPV); Oral Cancer; Oncogenic Viruses; Tobacco

\section{Introduction}

Oral cancer is the sixth most prevalent form of cancer malignancies, worldwide with squamous cell carcinoma being its most commonly detected histology. The incidence of oral cancer is on the rise due to the significant exposure to the risk factors, one of them being the oncogenic viruses [1]. The significant role of viruses in cancer was acknowledged in the later part of the last century, after the discovery of tumorigenic viruses in rodents. For the seminal discovery of these viruses, Rous was awarded the Nobel Prize in 1966 [1].

The most commonly reported and implicated oncogenic viruses as risk factors for oral cancer include human papillomavirus (HPV), hepatitis $\mathrm{C}$ viruses, herpes group viruses, and adenoviruses. Among these, HPV is the most exhaustively studied oncogenic virus along with the herpes virus. They are believed to be synergistically involved in the development of oral cancer [1].

The evidence of HPV as an etiological factor in oral carcinogenesis was first reported by Syrjanen., et al. in 1983 by microscopically observing the cytopathic effects of the virus in oral carcinoma. Their observations were confirmed by Chang., et al. in 1990 with the use of techniques such as in situ hybridization and polymerase chain reaction [1]. By 1997, the presence of HPV was reported in $31 \%$ (746 of 2380 cases) of the oral carcinoma samples. Subsequently, the studies conducted between 1998 and 2002 reported $12.4 \%$ (295 of 2388 cases) incidence of HPV DNA-positive oral carcinoma. The rise in the incidence of HPV-positive oral cancer cases was evidenced by the fact that $16 \%$ of HPV-positive cases were recorded between 1984 and 1989, which increased to $72 \%$ by 2000 $04[1]$. 
Due to the intense focus on detection of HPV in oral cancer, consistent reports of its prevalence have been noted, but with conflicting results. This difference in observations has been attributed to variation in the site of sample, patient population, methodology implemented and sample size [1]. Regardless, an increase in the prevalence of HPV-positive oral cancer over the past decade has indicated its potential role in the prediction of oral cancer lesions, in future [2].

This chapter highlights the recent advancements in the detection of HPV-positive cases of oral cancer. The methods have evolved from mere identification of HPV to being applied for specific and sensitive detection of the HPV serotypes, especially in the early stages of oral cancer [1]. The chapter also focuses upon emphasizing the steps and concepts being considered for updating the existent therapeutic measures to increase the survival rate and maximize the control of oral cancer with minimal effect on form and function of the diagnosed patients.

\section{HPV pathogenesis}

Awareness about the role of HPV as an etiological agent of oral cancer has increased the interest in acquiring more specific knowledge about HPV infections in the oral cavity. This section deals with an insight into the mode of infection, mechanism, and prognosis of HPV-positive oral cancer.

\section{HPV}

HPV has about 200 genotypes with marked tropism for the basal cells of the squamous epithelium. The HPV types have the distinction of infecting the squamous epithelium at different anatomical locations [1].

The open reading frame regions of the genome are divided into early (E; E1-7) and late (L; L1 and L2) regions. After infection, the expression of early genes occurs for encoding the proteins involved in the induction and regulation of viral replication. Viral capsid proteins are expressed by the late genes at a later stage of the infection [3].

\section{HPV infection in the oral cavity}

Research on oral cancer has been restricted to the study of buccal mucosa, the floor of mouth, vestibules, border and under the surface of the tongue and the gingiva. Samples from lips, tonsillar pillars and the base of the tongue are usually excluded. For the detection of infections, especially the asymptomatic HPV infection, the nonkeratinized surfaces are considered which have the collection of basal epithelial cells or nucleated cells.
The transmission of HPV infection is possible only by means of close contact. The common modes include sexual contact, vertical transmission during childbirth and autoinoculation. The clinical manifestations of HPV in the oral region include Heck disease, oral squamous papilloma, oral condyloma acuminatum, common warts, oral lichen planus, oral verrucous carcinoma, oral leukoplakia, and WHIM syndrome, apart from its role in oral squamous cell carcinoma (OSCC) [4]. The steps of HPV infection have been illustrated in figure 1.

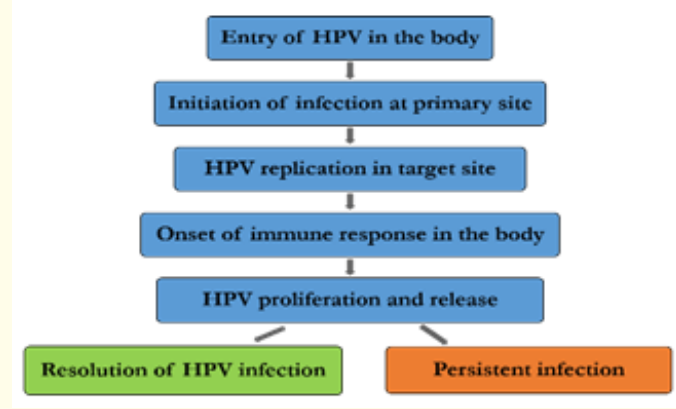

Figure 1: Steps of HPV infection [4].

\section{HPV types associated with oral cancer}

Based on its ability to transform the squamous epithelial cells, HPV has been classified as high-risk and low-risk types. Development of benign lesions is indicative of infection by low-risk HPV. High-risk HPV infection usually progresses to malignant lesions and cancer due to the loss of p53 function as result of expression of E7, hence are more commonly detected in cancer cells. Typically, the low-risk HPV exists episomally in the epithelial cells whereas the high-risk HPVs integrate within the genome or exist as episome in nuclei. This may be the reason for different manifestation upon HPV infection [1]. The most commonly detected HPV genotypes in oral cancer include HPV 16 and 18; however, the incidence of infection by HPV 33, 45 and 52 has been recorded in few cases $[5,6]$.

Upon infection, HPV is involved in the pathogenesis of benign lesions in the oral cavity, especially as verruca vulgaris, Heck's disease, and squamous papillomas. The affected regions of the oral cavity and common genotypes of HPV isolated have been represented in table $1[6]$. 
Table 1: Representation of types of HPV and the regions of oral cavity affected in humans.

\begin{tabular}{|l|c|c|c|c|}
\hline Region of oral cavity & High-Risk HPV & $\begin{array}{c}\text { Intermediate } \\
\text { Risk HPV }\end{array}$ & $\begin{array}{c}\text { Low-Risk } \\
\text { HPV }\end{array}$ & References \\
\hline Tongue & & HPV 31 & HPV 4 & Lee., et al. [7]. \\
Soft palate & Candotto., et al. [8]. \\
Lips & HPV 16, HPV 18 & HPV 33 & HPV 6 & Araujo., et al. [9]. \\
Floor of the mouth & HPV 35 & HPV 11 & Lupato., et al. [10]. \\
Cheek & HPV 52 & HPV 13 & Kouvousi., et al. [11]. \\
Gums & HPV 58 & HPV 32 & \\
Buccal mucosa & & & \\
Gingiva & & & & \\
Hard palate & & & \\
Mandibular and maxillar alveole & & & & \\
\hline
\end{tabular}

\section{HPV-induced carcinogenesis}

The malignancy of HPV after infection of the host cell is maintained by the activity of two proteins-E6 and E7. The mechanism of action of these proteins is represented in figure 2 [12].

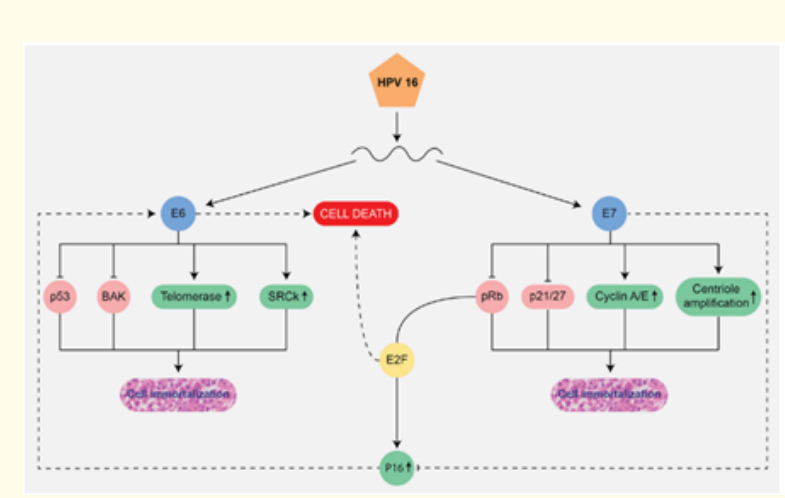

Figure 2: The activity of E6 and E7 proteins for maintaining malignancy of HPV infection [SRC, sarcoma] [12].

Cancer-inducing property of high-risk HPV has been identified to be specific to the E7 protein expressed during infection. But, the successful cancerous growth of cells is achieved only when p53 is deactivated, as it can detect the E7-related DNA insertion and cause disruption of cell division. Furthermore, the simultaneous activity of both the proteins i.e. E6 and E7 in a keratinocyte can accelerate the cancer growth by producing an uninhibited production of transcription factor-E2f [1].

\section{Prognosis}

The reports suggesting the ability of high-risk HPV to induce oral carcinogenesis has been on the rise. However, recent research indicates that these viruses have the ability to alter oral cancer phenotypes and outcome. The exploitation of this property has, therefore, become an important step to determine the appropriate prognosis and therapeutic options in oral cancer [1].

A study on cases of HPV-positive tongue cancer, the overall survival, and disease-free survival was better than those HPV-negative tongue cancer cases indicating the importance of consideration of HPV status while evaluating the prognosis of the case [13]. On the other hand, the high mortality rate was observed in HPV-positive patients in another study indicating poor prognosis as compared to HPV-negative patients [14]. In general, a clear picture of prognostic relevance of HPV-positive oral cancer has not yet been established due to the presence of contradictory reports. Some of the reports indicate better survival rates but these are diluted by reports of none or worst outcome [15].

The prognostic evaluation of the oral cancer is usually based on the clinical TNM classification (size, region, and spread of cancer). $\mathrm{T}$ indicates the size of a primary tumor, $\mathrm{N}$ indicates regional lymph nodes involvement and $\mathrm{M}$ describes metastasis. Accordingly, the bi- 
ological activity of oral cancer is classified into-highly, moderately and poorly differentiated. Other parameters of evaluation include the degree of keratinization, nuclear polymorphism, pattern and stage of invasion, which contributes to better prognosis in cancer cases [16]. The staging of cancer can also be described as stage 0-IV indicating the spread of cancer. Similarly, the cancer is also graded based on the level of abnormality into GX (undetermined), G1 (low grade), G2 (intermediate grade), G3 (high grade, poor differentiation) and G4 (high grade, undifferentiated) $[17,18]$.

The appropriate and accurate prognosis is essential for estimating survival rates and chances of recurrence of HPV infection. Due to poor prognosis, persistent HPV 16 DNA was detected in oral rinses post-treatment in a study by Rettig., et al. [19]. However, a recent study on HPV-associated OSCC indicates that the prognosis might not differ based on HPV positivity but there still is a need to consider HPV-associated OSCC for evaluating oral cancer [20].

\section{Diagnostic tests available for HPV detection}

The techniques for detection of HPV and related biomarkers vary substantially across different laboratories. This varies not only in design but also in their targets, including oncoproteins, HPV specific nucleic acids, cellular proteins or HPV-specific serum antibodies. For extensive implementation in the clinical arena, detection techniques should be cost-effective, accurate, and readily transferable to the routine diagnostic laboratories. Early detection is the key element for sufficient therapy of HPV-induced oral cancer. New technologies are emerging for early diagnosis of HPV-induced oral cancer through molecular analysis of cytologic smears or saliva samples. However, until today, a standard technique has not been specifically framed for detection of HPV-induced oral cancer.

\section{Conventional techniques}

Immunohistochemistry (IHC), in-situ hybridization (ISH), Southern blot hybridization are the conventional diagnostic tests employed in the detection of HPV in oral cancer [21-24] p16, a cellular protein strongly expressed in tumor cells, detected by IHC showed high sensitivity and less specificity. Therefore, p16 is a reliable surrogate biomarker in the prognosis of HPV-induced oral cancers [21] p16 IHC staining in histological grades of OSCC is represented in figure 3a-3e. Southern blot technique and ISH are the molecular methods used in the detection of HPV-induced oral cancer. Southern blot hybridization is the sophisticated method for the detection of DNA sequences in HPV-induced oral cancer [24].
However, the technique was unable to visualize histomorphologic characteristics of HPV positive samples. ISH is another sensitive technique which aids in detection, typing, and localization of viral DNA in paraffin-embedded tissues. It is a signal amplification technique that uses biotinylated HPV specific DNA probes that aids in the detection of DNA sequences in HPV positive samples [23,25]. Table 1 represents different techniques and detection targets for screening HPV-induced oral cancer.

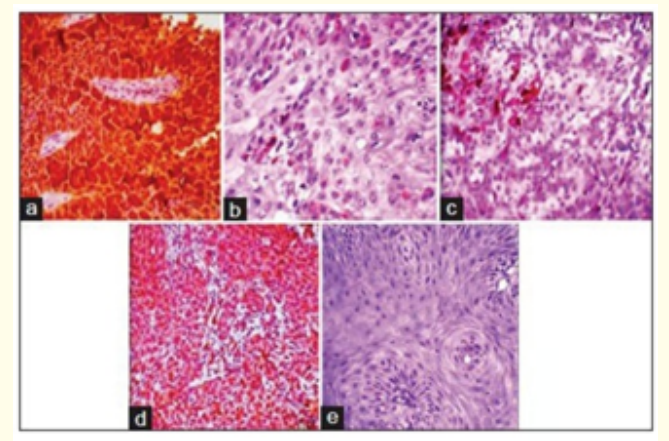

Figure 3a-e: p16 immunohistochemistry staining in histological grades of oral squamous cell carcinoma with the positive control. a) Positive control slide of cervical cancer, (b) dispersed single cell staining in well-differentiated oral squamous cell carcinoma, (c) Patchy staining in moderately differentiated oral squamous cell carcinoma, (d) diffuse staining in poorly differentiated oral squamous cell carcinoma, (e) No staining [21].

\section{Recent established techniques}

Along with conventional techniques, several lab-based techniques are widely used in the detection of HPV-induced oral cancer. Such commonly established techniques include Hybrid capture Digene ${ }^{\circledR}$ test, Polymerase chain reaction (PCR), Flow cytometer analysis and quantitative polymerase chain reaction (qPCR). Hybrid capture Digene ${ }^{\circledR}$ test aids in the detection of low-risk and high-risk HPV in oral cancer [26]. PCR is the other standard and sensitive technique in the detection of HPV DNA in oral cancer (Figure 3f). High sensitivity, type-specific, and formalin-fixed paraffin-embedded (FFPE) tissue acceptances are the advantages of PCR technique [27]. However, the technique is laborious and lacks quantitation of viral DNA to assess the role of viral load in disease pathogenesis. While the novel qPCR technique allows rapid detection and quan- 
titation of HPV. The quantitation of DNA is more using qPCR technique. The real-time qPCR technique is able to differentially detect and quantify 4 types of $\operatorname{HPV}(6,11,16$, and 18) [28]. Increased viral loads are found in high-risk (HPV-16, HPV-18) than low-risk (HPV-6, HPV-11) HPV-induced oral cancer. Both the quantitative E6 DNA and E6 RNA PCR are highly accurate in the diagnosis of HPV-induced oral cancer [22]. Moreover, the detection of E6/E7 mRNA is currently considered as the gold standard technique in prognosticating HPV-induced oral cancer. The presence of E6/E7 mRNA not only indicates the presence of HPV but also states that it is transcriptionally active. Unfortunately, it is the most challenging technique restricted to research laboratories [29]. Flow cytometer analysis is the other detection technique employed in the detection of E6, E7 mRNA expression in HPV-induced oral cancer (Figure 3g). E6, E7 mRNA probes labelled with PD-L1 Ab are used in the detection of cancer [30]. Table 1 represents different techniques and detection targets for screening HPV-induced oral cancer.

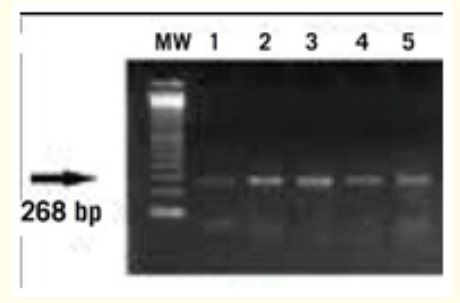

Figure 3F: Electrophoresis in 2\% agarose gel stained with ethidium bromide; Showing $\beta$-globin DNA amplification (268 bp). HPV detection in floor of mouth squamous cell carcinoma by PCR amplification [27].

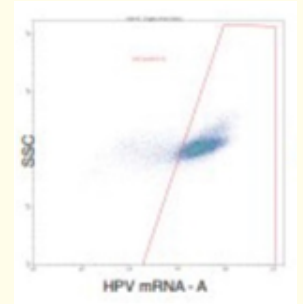

Figure 3G: Showing HPV E6, E7 mRNA overexpression in oral cancer samples. Comparison of HPV E6, E7 mRNA and p16

Expression in Oral Cancer Samples Using Flow Cytometry and Immunohistochemistry [30].
Research on biomarkers

Although several techniques identified HPV DNA sequences in HPV-induced cancer, this alone is not adequate to drive a causal association from a molecular perspective. Hence studies have targeted on the expression of two HPV viral oncoproteins such as E6 and E7 DNA and RNA for classification of an HPV-caused oral cancer. E6 and E7 play a significant role in tumorigenesis by dysregulating the tumor suppressor genes p53 and Rb. Hence, E6 and E7 DNA and RNA might be prognostic parameters in the detection of HPV-induced oral cancers. However, a little research has been done with an emphasis on E6/E7 DNA and RNA.

A study conducted by Tsimplaki., et al. [31] and Kouvousi., et al. [11] found high-risk HPV E6/E7 mRNA expression in OSCC which suggested the pathogenic role of HPV in oral cancer. The studies used PapilloCheck ${ }^{\circledR}$ HPV genotyping assay for the identification of 24 types of HPV and finally found HPV 16 was the most prevalent genotype both by DNA and RNA assays. Real-time nucleic acid sequence-based amplification and NucliSENS ${ }^{\circledR}$ EasyQ ${ }^{\circledR}$ assay were performed for the qualitative detection of E6/E7 oncoproteins $[11,31]$.

A study conducted by Wong., et al. [32] found that HPV 16 E6/ E7 seropositivity was strongly associated with oral cancer The study performed Glutathione S Transferase (GST)-capture ELISA to determine the prevalence of HPV seropositivity among healthy as well as oral cancer patients.

In addition to E6 and E7, microRNAs also function as oncogenes and tumor suppressors. Loss of tumor suppressive miRs along with upregulation of oncogenic miRs in host cells have been reported in HPV-induced malignancies [33]. It was reported that proactive patterns implicate the dysregulation of multiple miRs in HPV-induced cervical and oropharyngeal cancer [34]. Hence, the role of microRNAs in HPV-induced oral cancers can be investigated in future studies. A study conducted by Shi., et al. [35], performed fluorescence (ISH) technique to find the expression of microRNA in archived (FFPE) oral cancer tissues. Locked nucleic acid-based probes labeled with digoxigenin at $5^{\prime}$ end was used to detect the microRNA expression.

Although the conventional and novel detection techniques have advantages and disadvantages; qPCR can be considered as the established technique in the detection of HPV-induced oral cancer. 


\begin{tabular}{|l|c|}
\hline Screening techniques & Target \\
\hline Immunohistochemistry [18] & Protein-p16 \\
\hline Southern blot hybridization [24] & HPV DNA \\
\hline In situ hybridization [23] & HPV DNA \\
\hline Hybrid capture Digene ${ }^{\circledR}$ test [26] & $\begin{array}{c}\text { Low-risk and high-risk } \\
\text { DNA }\end{array}$ \\
\hline Polymerase chain reaction [36] & HPV E6 DNA \\
\hline $\begin{array}{l}\text { Quantitative polymerase chain } \\
\text { reaction [22] }\end{array}$ & HPV E6 DNA and RNA \\
\hline Flow cytometer analysis [30] & HPV E6, E7 mRNA \\
\hline
\end{tabular}

Table 2: Techniques established in screening HPV-induced oral cancer.

\begin{tabular}{|c|c|c|}
\hline $\begin{array}{l}\text { Screening } \\
\text { techniques }\end{array}$ & Advantages & Disadvantages \\
\hline $\begin{array}{l}\text { Microscopic } \\
\text { evaluation }\end{array}$ & $\begin{array}{l}\text { Low cost and serves as a } \\
\text { standard for other HPV } \\
\text { tests }\end{array}$ & $\begin{array}{l}\text { Not specific when } \\
\text { used alone }\end{array}$ \\
\hline $\begin{array}{l}\text { Polymerase } \\
\text { chain reaction }\end{array}$ & Highly sensitive & $\begin{array}{l}\text { Unable to differen- } \\
\text { tiate biologically } \\
\text { between relevant } \\
\text { and irrelevant infec- } \\
\text { tions }\end{array}$ \\
\hline $\begin{array}{l}\text { Quantitative } \\
\text { polymerase } \\
\text { chain reaction }\end{array}$ & $\begin{array}{l}\text { Highly sensitive and } \\
\text { specific }\end{array}$ & $\begin{array}{l}\text { Requires fresh } \\
\text { frozen tissue and } \\
\text { technically chal- } \\
\text { lenging }\end{array}$ \\
\hline $\begin{array}{l}\text { in situ hybrid- } \\
\text { ization }\end{array}$ & $\begin{array}{l}\text { Highly specific, easy to } \\
\text { use }\end{array}$ & $\begin{array}{l}\text { Low sensitivity, dif- } \\
\text { ficult to interpret }\end{array}$ \\
\hline $\begin{array}{l}\text { Immunohisto- } \\
\text { chemistry }\end{array}$ & $\begin{array}{l}\text { Low cost, highly sensi- } \\
\text { tive, widely used in } \\
\text { most laboratories }\end{array}$ & $\begin{array}{l}\text { Not highly specific } \\
\text { and should be cor- } \\
\text { rectly interpreted }\end{array}$ \\
\hline $\begin{array}{l}\text { p16 + Other } \\
\text { methods (PCR } \\
\text { and ISH) }\end{array}$ & $\begin{array}{l}\text { Combination of highly } \\
\text { sensitive p16 with other } \\
\text { specific method in- } \\
\text { creases the probability } \\
\text { of HPV detection }\end{array}$ & Increased cost \\
\hline $\begin{array}{l}\text { Serological } \\
\text { test }\end{array}$ & Very easy & $\begin{array}{c}\text { Not related to viral } \\
\text { DNA }\end{array}$ \\
\hline
\end{tabular}

Table 3: Comparison of different methods of detecting Human papillomavirus in cancer patients [29].

\section{Therapy/treatment for HPV infection}

Surgery, radiotherapy, and chemotherapy individually or in combination are the treatment modalities available currently to treat HPV-induced oral cancer [37]. Alike other HPV-induced can- cers, surgery and/or radiotherapy are the choice of treatment in the primary (I and II) stages of oral cancer, which usually assures a permanent cure. Combination of surgery, radiotherapy or chemotherapy is used for the treatment of the $3^{\text {rd }}$ or $4^{\text {th }}$ stage of oral cancer [38].

\section{Current treatment modalities}

\section{Surgery}

Surgery is the first-line choice of treatment in early stages of cancer. However, favorable oncologic outcomes with advances in chemotherapy and radiotherapy have shifted choice of treatment away from surgery [39]. Transoral laser microsurgery, transoral robotic surgery, and trans-oral surgery are the currently used procedures in the treatment of cancers. With advancements in all these minimally invasive surgeries, these can be effectively employed in the treatment of HPV-induced oral cancers [39].

\section{Chemotherapy}

Cisplatin is the most commonly employed chemotherapeutic agent in the treatment of cancers. Cisplatin has a good prognostic outcome with 3-year survival rate of $90 \%$. Cisplatin, cis-Diammineplatinum (II) dichloride, is a DNA intercalator that targets cells replicating at a higher rate binds to guanine residues and forms crosslinks between two DNA stands which eventually leads to apoptosis. Studies found that HPV-induced cancer patients showed an increased response to platinum-based chemotherapy than those of HPV-negative counterparts. Hence these can be effectively used in the management of HPV-induced oral cancer. Xerostomia, neurotoxicity dysphagia and renal failure are the few side effects of chemotherapy. Moreover, this treatment regimen serves as a standard method for organ preservation. Other commonly used platinum-based chemotherapeutic agents include taxanes such as docetaxel and paclitaxel, carboplatin; 5-fluorouracil and methotrexate. All these drugs showed promising results in the treatment of HPV-induced head and neck cancers. Therefore, all these agents can be exploited in the treatment of HPV-induced oral cancer [39].

\section{Radiotherapy}

Radiotherapy is the other conventional treatment employed in the treatment of cancer. Radiotherapy induces breakdown of double-stranded DNA, reduces cell viability, increases cell cycle arrest and leads to the death of tumor cells. Intensity-modulated radiotherapy, an advancement in radiotherapy, delivers radiation only to carcinogenic cells and simultaneously spares and reduces 
dosage to non-carcinogenic cells. Research has found that transoral robotic surgery followed chemotherapy and radiation can be effectively employed to both HPV-induced and HPV non-induced cancers to control disease pathogenesis [40]. Therefore, this combined treatment can be effectively implemented in the treatment of HPV-induced oral cancers. In case of radiotherapy, oral care should be specifically important, since there is a liability, particularly to mucositis, xerostomia, and osteonecrosis. However, advancement in therapeutic approach, overall 5-year survival rate using these techniques in head and neck cancer patients was only $50 \%$. Dysphagia, lymphedema, fibrosis and cardiovascular disease are the other side effects associated with radiotherapy [38].

\section{Management of HPV-induced oral cancer: The debate}

Research has reported that HPV expression showed high response rate to conventional chemotherapy, radiotherapy, and radiochemotherapy $[38,39,42]$. Studies conducted on patients with OPSCC reported that overall 3-year survival rate of HPV-associated malignancies was about $75 \%$ as compared to $50 \%$ of those with HPV-unassociated OPSCC. Correspondingly, studies conducted on HPV-induced head and neck cancer patients showed drop of 50\% in recurrences, $40 \%$ drop in death rates and lower incidence of metastases than those with HPV-negative counterparts. Overall, HPV-induced cancers reported high therapeutic response rate to chemotherapy and radiotherapy with respect to intervention and survival rate. Currently, no clinical guidelines have been set in distinguishing treatment approaches between HPV-induced and other factors induced oral cancers. However, at present, researchers are understanding the need for differentiating the therapeutic approaches between HPV-positive and HPV-negative tumors due to its discrete disease etiologies [38].

Since the HPV was associated in tissues of head and neck cancers, the study of molecular pathways in HPV-induced head and neck cancers has reaped considerable attention [38]. Similarly, current research is being targeted on molecular therapy with monoclonal antibodies and gene therapy to treat HPV-induced oral cancers. Unlike surgery, chemotherapy, and radiotherapy, this targeted molecular therapy has nonexistent or limited adverse effects on normal cells of the body [38].

\section{Novel therapeutic approaches}

Molecular targeted therapy, an established treatment modality in cervical, head and neck and oropharyngeal cancers, targets pro- teins and molecules selectively expressed by tumor cells. These include signal transduction molecules, hormones, growth factors and their receptors, apoptosis-related molecules, angiogenesis-related factors, oncogenes, and inhibitors of cell motility, proteolysis, and invasion [43]. In HPV-induced oral cancers, the HPV infection leads to the expression of E6 and E7 oncoproteins. Along with these, E1 and E2 are the other oncoproteins expresses at a high level before viral genome integration. Therefore, the suppression of these viral oncoproteins with the use of medicines/therapies especially molecular targeted therapies can be an effective technique in controlling the growth of the tumor or the cancer cells.

\section{HPV vaccination for oral cancer prevention}

Targeting HPV plays a critical role in the prevention of HPV-induced oral cancer. This can be achieved through prophylactic vaccines. Development of prophylactic vaccines induces the cytolytic immune response towards the cancer cells expressing the oncogenes to prevent the establishment of HPV infection, even at the advanced stage. Prophylactic vaccines also stimulate the immune system to produce neutralizing antibodies former or on exposure to high-risk HPV to prevent the formation of persistent infection. These neutralizing antibodies bind to the neutralizing epitopes on viral surfaces and blocks entry of the virus into the host cells. This mechanism has led to the development of bivalent and quadrivalent vaccines to protect against high-risk HPV subtypes [44,45].

\section{History}

biHPV (GSK, 2009) and qHPV vaccines (Merck, 2006) are the two vaccines approved by FDA to prevent HPV-induced cancers. The biHPV vaccine consists of AS04, a modified diphosphoryl lipid that activates cellular and humoral immune responses with the aluminum hydroxide in an adsorbed form as a general adjuvant. The safety and efficacy of the biHPV vaccine were confirmed through clinical trials (I, II and III) in the subsequent age groups: 10 - 14 years, 15 - 25 years, 10 - 25 years, and 15 - 55 years. The other virus-like particle (VLP)-based vaccine (qHPV) developed was HPV L1 prophylactic vaccine, which has the same structure as HPV 16 and has four viral antigens produced from the yeast Saccharomyces cerevisiae expression system. Purified VLPs are adsorbed on aluminum hydroxyphosphate sulfate and formulated with sodium borate, sodium chloride, L-histidine, polysorbate-80, and water for injection. Then, the produced antigens are combined with the adjuvant to form the qHPV vaccine. Although biHPV and qHPV are 
effective in preventing HPV-induced cancer, these reported various adverse effects such as fever, local reaction, erythema, menstrual problems, headache, and malaise. Overall, these vaccines reported $53.9 / 100,000$ of adverse effects [46]. Therefore, this led to the development of new HPV vaccines.

\section{Current}

Currently, three HPV vaccines have been included in national immunization programs with an objective of preventing oral cancer. The three vaccines-Cervarix, Gardasil, and Gardasil 9 are approved by the FDA to prevent HPV-induced oral cancer. Cervarix (GSK) is a bivalent vaccine that prevents infection with HPV subtypes 16 and 18, Gardasil is a quadrivalent vaccine that prevents infection with HPV subtypes 6,11,16, and 18. While, Gardasil 9 is a 9-valent vaccine that prevents infection with HPV subtypes 6, 11, 16 , and $18,31,33,45,52$, and 58 . However, both bivalent and quadrivalent vaccines are effective in preventing both HPV-16 and HPV18 induced oral cancer. CDC recommends all the adolescents ages 11 or 12 years must get HPV vaccination. The vaccines can also be started at 9 years; however, should be completed before 13 years. All the children and adults can also receive vaccination between 13 and 26 years if they have not received the HPV vaccine earlier. The vaccines can also be given before the individuals become sexually active. The children aged between 11 and 12 years should receive two doses of HPV vaccine at least six months apart from previously recommended three doses. Children and adults who start the vaccination, i.e., 15 to 26 years, still requires three doses of HPV vaccine to protect against oral cancer [46].

\section{Vaccine composition}

All the three vaccines comprise synthetically made virus-like particles (VLPs) of the L1 epitope. Of all three vaccines, Cervarix contains least antigenic concentration and has AS04, advanced adjuvant to boost immunogenicity.

Gardasil 9 has two times more the antigenic load and the aluminium load compared to Gardasil. Gardasil 9 has high concentrations of L1 VLPs for HPV 16 as well as 18 to induce antibody responses that are superior to Gardasil [45].

\section{Future}

Although the currently available HPV vaccines are effective in preventing the HPV-induced oral cancer, they are not extremely effective in clearing pre-existing infections [46]. Therefore, the launching of therapeutic vaccines is the need of an hour to treat established infections. Therapeutic vaccines trigger cell-mediated immune responses (T-helper type 1 cells) and cytotoxic T-cells (derived from CD4+ and CD8+ T-cells) for spontaneous clearance and slow-progression of pre-existing HPV infections $[47,48]$. The HPV E6 and E7 are the early genes that play a major role in the disruption of the cell cycle in premalignant and malignant lesions. Also, these genes are expressed at the very high level, therefore signify ideal and potential targets to produce therapeutic vaccines for established HPV-induced cancer/tumors. The therapeutic vaccines should target oncoproteins (E6 and E7) to induce strong cytotoxic lymphocyte and tumor-specific T-cell type 1 responses to invade malignant lesions $[48,49]$. Other oncoproteins (HPV E1 and E2) that express at a high level before viral genome integration can also be targeted with therapeutic vaccines to prevent HPV-induced cancer [48].

Several therapeutic vaccine strategies such as live-vector vaccines (bacterial and viral), subunit vaccines (peptides, proteinbased, plant peptide/protein based), nucleic acid (DNA, RNA replicon-based DNA), and cell-based vaccines (dendritic, adoptive cell transfer) and other combinatorial approaches have been investigated for the development of therapeutic vaccines. However, till today no therapeutic vaccines are approved for human use. Although, with a thorough understanding of HPV life cycle, carcinogenesis, molecular mechanisms of infection, tumor biology, tumor microenvironment and immune response mechanisms, the fewer clinical trials have been conducted for the development of HPV therapeutic vaccine [48]. Hence, future studies should be conducted focusing on therapeutic vaccines to prevent pre-existing HPV-induced oral cancer.

\section{Conclusion}

Although the research has been focused on the role of HPV in oral cancer. Still, there are gaps to be bridged regarding the identification of potential biomarkers, diagnosis, detection techniques, and therapeutic targets in HPV-induced oral cancer. Following are the few future recommendations including gaps in the research that need to be addressed for a better understanding of HPV-induced oral cancer: 
- $\quad$ The prevalence, incidence, and trends of HPV-induced cancers by demographic factors at different anatomical sites is wellknown. While the literature available for HPV-induced oral cancer is scarce. Therefore, research on prevalence, incidence, and trends benefits the oral pathologists in better identifying the patients with HPV-induced oral cancer, diagnose the disease at early stages, and upsurges the overall survival rate.

- Along with traditional risk factors-alcohol consumption, smoking, tobacco consumption, recently HPV is considered as the main etiological factor in oral cancers. However, the exact pathogenetic role and host immune response in HPV-induced oral cancers is not yet clear. Therefore, future studies emphasizing on these features aids the accurate differentiation of HPV-positive from HPV-negative patients, development of new therapies and prevention of malignant/premalignant lesions at the early stages.

- Currently available techniques in the literature are not only illequipped for their immediate use as diagnostic aids but also restricted only in diagnosing the advanced stage of cancer. Hence, research is required to develop a simple, efficient, fast, portable and at a cost-effective diagnostic tool for the detection of HPV-induced oral cancer even early stages. The detection techniques might also influence the prevalence of HPVinduced oral cancer. Thus, a standardized technique needs to be framed for the detection of HPV-induced oral cancer.

- The combination of HPV16 DNA detection or immunostaining (p16) with E6/E7 antibodies signifies the most clinically reliable surrogate markers for the better identification of HNSCC patients. This can also be evaluated in HPV-induced oral cancer patients for multimodal approach of diagnosis.

- HPV DNA load quantification and expression of E6 and E7 are the promising biomarkers in the prediction of HPV-induced cervical, and head and neck cancers. Therefore, targeting these can also serve as a potential biomarker in HPV-induced oral cancer.

- $\quad$ Along with qRT-PCR other high throughput screening techniques such as microarray and next-generation sequencing can also be used in the detection of HPV-induced oral cancer. The implementation of microarray technique aids in the accurate detection of HPV subtypes. While the introduction of next-generation sequencing gives comprehensive information about the HPV-induced tumors. Also, these provide detailed information about disrupted genes and pathways, genomic HPV integration sites, and distinct genetic as well as epigenetic alterations in HPV-induced cancers.
- $\quad$ There are many techniques available in the detection of proteomic and genomic biomarkers, none of them are standardized techniques; therefore, in future, studies can be conducted on identifying metabolomic biomarkers including lipids, carbohydrates, enzymes, and metabolites in the detection of HPVinduced oral cancer.

- Available prophylactic vaccines are not effective in preventing pre-existing HPV infections. Hence, the research can be emphasized to develop therapeutic vaccines to generate cellmediated immune response against HPV oncoproteins E6/E7.

- Public education programs can be implemented especially for young adults regarding the role of HPV and unsafe sexual practices for the prevention of HPV-induced oral cancer.

\section{Bibliography}

1. Metgud R., et al. "Role of viruses in oral squamous cell carcinoma”. Oncology Reviews 6.2 (2012): e21.

2. Lafaurie GI., et al. "Human papilloma virus: An etiological and prognostic factor for oral cancer?". Journal of Investigative and Clinical Dentistry 9.2 (2018): e12313.

3. Zheng ZM and Baker CC. "Papillomavirus genome structure, expression, and post-transcriptional regulation". Frontiers in Bioscience 11 (2006): 2286-2302.

4. Rautava J and Syrjänen S. Human papillomavirus infections in the oral mucosa". Journal of the American Dental Association 142.8 (2011): 905-914.

5. Kingma DW., et al. "HPV genotype distribution in oral and oropharyngeal squamous cell carcinoma using seven in vitro amplification assays". Anticancer Research 30.12 (2010): 50995104.

6. Michaud DS., et al. "High-risk HPV types and head and neck cancer". International Journal of Cancer 135.7 (2014): 16531661.

7. Lee LA., et al. "Human papillomavirus-16 infection in advanced oral cavity cancer patients is related to an increased risk of distant metastases and poor survival". PloS one 7.7 (2012): e40767.

8. Candotto V., et al. "HPV infection in the oral cavity: epidemiology, clinical manifestations and relationship with oral cancer". Oral and Implantology 10.12 (2017): 209-220.

9. Araújo MV., et al. "Prevalence of human papillomavirus (HPV) in Belem, Para State, Brazil, in the oral cavity of individuals without clinically diagnosable injuries". Cadernos de saude publica 30.5 (2014): 1115-1119. 
10. Lupato V., et al. "Prevalence and Determinants of oral human papillomavirus infection in 500 young adults from Italy". PloS one 12.1 (2017): e0170091.

11. Kouvousi M., et al. "Prevalence of human papillomavirus in 45 Greek patients with oral cancer". Journal of oncology (2013).

12. Boulet G., et al. "Human papillomavirus: E6 and E7 oncogenes". The International Journal of Biochemistry and Cell Biology 39.11 (2007): 2006-2011.

13. Attner P., et al. "Human papillomavirus and survival in patients with base of tongue cancer". International Journal of Cancer 128.12 (2011): 2892-2897.

14. Saghravanian N., et al. "Evaluation of the prevalence rate and the prognostic effect of human papilloma virus infection in a group of patients with oral cavity squamous cell carcinoma". Iranian Journal of Cancer Prevention 9.3 (2016): e3998.

15. Hübbers CU and Akgül B. "HPV and cancer of the oral cavity". Virulence 6.3 (2015): 244-248.

16. Akhter M., et al. "A study on histological grading of oral squamous cell carcinoma and its co-relationship with regional metastasis". Journal of Oral and Maxillofacial Pathology 15.2 (2011): 168.

17. National Cancer Institute. Cancer staging (2018).

18. National Cancer Institute. Tumor grade (2018).

19. Rettig EM., et al. "Prognostic implication of persistent human papillomavirus type 16 DNA detection in oral rinses for human papillomavirus-related oropharyngeal carcinoma". JAMA Oncology 1.7 (2015): 907-915.

20. Kim SM., et al. "Identification of human papillomavirus (HPV) subtype in oral cancer patients through microarray technology". European Archives of Oto-Rhino-Laryngology 275.2 (2018): 535-543.

21. Patil S., et al. "Analysis of human papilloma virus in oral squamous cell carcinoma using p16: An immunohistochemical study". Journal of International Society of Preventive and Community Dentistry 4 (2014): 61-66.

22. Pannone G., et al. "Evaluation of a combined triple method to detect causative HPV in oral and oropharyngeal squamous cell carcinomas: p16 Immunohistochemistry, Consensus PCR HPV-DNA, and In Situ Hybridization". Infectious agents and cancer 7 (2012): 4.
23. Van Rensburg EJ., et al. "Detection of human papillomavirus DNA with in situ hybridisation in oral squamous carcinoma in a rural black population". South African Medical Journal (1995): 85.

24. Chang KW., et al. "High prevalence of human papillomavirus infection and possible association with betel quid chewing and smoking in oral epidermoid carcinomas in Taiwan". Journal of Medical Virology 28 (1989): 57-61.

25. Chang F., et al. "Detection of human papillomavirus (HPV) DNA in oral squamous cell carcinomas by in situ hybridization and polymerase chain reaction". Archives of Dermatological Research 282 (1990): 493-497.

26. Vidal AKdL., et al. "HPV detection in oral carcinomas". Jornal Brasileiro de Patologia e Medicina Laboratorial 40 (2004): 2126.

27. Simonato LE., et al. "HPV detection in floor of mouth squamous cell carcinoma by PCR amplification". Jornal Brasileiro de Patologia e Medicina Laboratorial 52 (2016): 43-49.

28. Seaman WT., et al. "Detection and quantitation of HPV in genital and oral tissues and fluids by real time PCR". Virology Journal 7 (2010): 194.

29. Pérez Rizo A. "New epidemiologic trends in human papillomavirus-related oropharynx cancer in Girona: a cross-sectional study [Thesis]". (2016).

30. Carrasco Y., et al. "Comparison of HPV E6, E7 mRNA/PD-L1 assay using flow cytometry and p16 IHC expression in oral cancer samples". Journal of Clinical Oncology 36.5 (2018): 114114.

31. Tsimplaki E., et al. "Prevalence and expression of human papillomavirus in 53 patients with oral tongue squamous cell carcinoma”. Anticancer research 34 (2014): 1021-1025.

32. G Ha K., et al. "Seropositivity of HPV 16 E6 and E7 and the risk of oral cancer". Oral diseases 20 (2014): 762-767.

33. Shah SS., et al. "Current technologies and recent developments for screening of HPV-associated cervical and oropharyngeal cancers". Cancers 8 (2016): 85.

34. Wise-Draper TM and Wells SI. "Papillomavirus E6 and E7 proteins and their cellular targets". Frontier Bioscience 13 (2008): 1003-1017. 
35. Shi Z., et al. "Fluorescence in situ hybridization for microRNA detection in archived oral cancer tissues". Journal of Oncology (2012).

36. Miller CS., et al. "Detection of HPV DNA in oral carcinoma using polymerase chain reaction together with in situ hybridization". Oral Surgery, Oral Medicine, Oral Pathology and Oral Radiology 77 (1994): 480-486.

37. Lai K., et al. "Differences in survival outcome between oropharyngeal and oral cavity squamous cell carcinoma in relation to HPV status". Journal of Oral Pathology and Medicine 46 (2017): 574-582.

38. Whang SN., et al. "Recent progress in therapeutic treatments and screening strategies for the prevention and treatment of HPV-associated head and neck cancer". Viruses 7 (2015): 5040-5065.

39. Nichols AC., et al. "Early-stage squamous cell carcinoma of the oropharynx: radiotherapy vs. trans-oral robotic surgery (ORATOR)-study protocol for a randomized phase II trial”. BMC cancer 13 (2013): 133.

40. Haedicke J and Iftner T. "Human papillomaviruses and cancer". Radiotherapy and oncology 108 (2013): 397-402.

41. Bol V and Grégoire V. "Biological basis for increased sensitivity to radiation therapy in HPV-positive head and neck cancers". BioMed Research International (2014).

42. Elrefaey S., et al. "HPV in oropharyngeal cancer: the basics to know in clinical practice". Acta Otorhinolaryngologica Italica 34 (2014): 299-309.

43. Hamakawa H., et al. "Basic evidence of molecular targeted therapy for oral cancer and salivary gland cancer". Head and Neck 30 (2008): 800-809.

44. Tumban E., et al. "A pan-HPV vaccine based on bacteriophage PP7 VLPs displaying broadly cross-neutralizing epitopes from the HPV minor capsid protein, L2". PloS One 6 (2011): e23310.

45. Harper DM., et al. "HPV vaccines-a review of the first decade". Gynecologic Oncology 146 (2017): 196-204.

46. Kim SM. "Human papilloma virus in oral cancer". Journal of the Korean Association of Oral and Maxillofacial Surgeons 42 (2016): 327-336.

47. Van Der Burg SH and Melief CJ. Therapeutic vaccination against human papilloma virus induced malignancies". Current Opinion in Immunology 23 (2011): 252-257.
48. Chabeda A., et al. "Therapeutic vaccines for high-risk HPV-associated diseases". Papillomavirus Research (2017).

49. Morrow MP., et al. "Human papillomavirus therapeutic vaccines: targeting viral antigens as immunotherapy for precancerous disease and cancer". Expert Review of Vaccines 12 (2013): 271-283.

\section{Assets from publication with us}

- Prompt Acknowledgement after receiving the article

- Thorough Double blinded peer review

- Rapid Publication

- Issue of Publication Certificate

- High visibility of your Published work

Website: www.actascientific.com/

Submit Article: www.actascientific.com/submission.php

Email us: editor@actascientific.com

Contact us: +919182824667 